\title{
Successive linear approximation for finite elasticity
}

\author{
I-SHIH LIU, ROLCI A. CIPOLATTI and MAURO A. RINCON \\ Instituto de Matemática, Universidade Federal do Rio de Janeiro \\ 21945-970 Rio de Janeiro, RJ, Brasil \\ E-mails: liu@im.ufrj.br / cipolatti@im.ufrj.br / rincon@dcc.ufrj.br
}

\begin{abstract}
A method of successive Lagrangian formulation of linear approximation for solving boundary value problems of large deformation in finite elasticity is proposed. Instead of solving the nonlinear problem, by assuming time steps small enough and the reference configuration updated at every step, we can linearize the constitutive equation and reduce it to linear boundary value problems to be solved successively with incremental boundary data. Moreover, nearly incompressible elastic body is considered as an approximation to account for the condition of incompressibility. For the proposed method, numerical computations of pure shear of a square block for Mooney-Rivlin material are considered and the results are compared with the exact solutions.
\end{abstract}

Mathematical subject classification: Primary: 65C20; Secondary: 74B20.

Key words: large deformation, nonlinear elastic materials, successive Lagrangian formulation, boundary value problem, incremental method, numerical computation.

\section{Introduction}

The constitutive equation of a solid body is usually expressed relative to a preferred reference configuration which exhibits specific material symmetries such as isotropy. The constitutive functions are generally nonlinear. Therefore, for large deformations, it leads to a system of nonlinear partial different equations for the solution of boundary value problems. The problems are usually formulated in Lagrangian coordinates in the preferred reference configuration, and 
numerical methods involve solving nonlinear systems as well as having other difficulties, such as the boundary conditions may involve the deformed state which depends on the solutions themselves. Alternatively, we are proposing a different method for solving boundary value problem of large deformation in a successive Lagrangian formulation of linear approximation.

Roughly speaking, the method of successive linear approximation is similar to the Euler method for solving differential equations, i.e., successively at each state, the tangent is calculated and projected to a neighboring state. In other words, the constitutive equations are calculated at each state which will be regarded as the reference configuration (updated Lagrangian formulation) for the next state, and assuming the deformation to the next state is small, the updated constitutive equations are linearized. In this manner, it becomes a linear problem just like the problems in linear elasticity from one state to the next state with incremental small deformations.

For numerical computation of large deformations, "incremental methods" have been widely discussed $([4,7,6])$. The problems are usually formulated with domains in the initial reference configuration, i.e., in "total" Lagrangian formulation. This is the essential difference from the method proposed in this paper in which at each step the reference configuration is updated in the "successive" Lagrangian formulation. Although the two approaches are mathematically equivalent, the basic equations and numerical treatment of boundary conditions are quite different. From the examples of pure shear, some advantages of the present approach are discussed.

\section{Updated reference configuration}

Let $\kappa_{r}$ be the preferred reference configuration of an elastic body $\mathbb{B}$, and

$$
\boldsymbol{x}=\chi(X, t), \quad X \in \kappa_{r}(\mathbb{B}),
$$

be the deformation of the body $\kappa_{r}(\mathbb{B})$ at time $t$. Let $F(X, t)$ be the deformation gradient and the Cauchy stress $T(X, t)$ be given by the constitutive equation

$$
T=\mathbb{F}_{\kappa_{r}}(F) \text {. }
$$


It is well-known that the constitutive model based on the linear constitutive equation, Hooke's law, does not satisfy the principle of material frame-indifference, and it can only be regarded as an approximation of some nonlinear model for small deformations (see [3, 2]). Therefore, in order to consider large deformations, the constitutive function $\mathbb{F}_{\kappa_{r}}$ is generally a nonlinear function of the deformation $F$.

Let $\kappa_{t_{0}}$ be the deformed configuration at time $t_{0}$. The deformation

$$
\xi=\chi\left(X, t_{0}\right)
$$

from $\kappa_{r}$ to $\kappa_{t_{0}}$ need not be small. Let

$$
F_{0}=F\left(X, t_{0}\right)=\nabla_{X} \boldsymbol{\xi}, \quad T_{0}=T\left(X, t_{0}\right),
$$

be the deformation gradient with respect to configuration $\kappa_{r}$ and the Cauchy stress at $t_{0}$ respectively.

Now, consider a deformation from $\kappa_{t_{0}}$ to the configuration $\kappa_{t}$ at time $t>t_{0}$ such that the displacement vector

$$
\boldsymbol{u}=\chi(X, t)-\boldsymbol{\xi}, \quad H=\nabla_{\xi} \boldsymbol{u},
$$

where $H$ is the displacement gradient with respect to the updated reference configuration $\kappa_{t_{0}}$ (emphasize, not $\kappa_{r}$ ). Then

$$
F=F(X, t)=\nabla_{X} \xi+\nabla_{\xi} \boldsymbol{u} \nabla_{X} \boldsymbol{\xi},
$$

or

$$
F=(I+H) F_{0} .
$$

Therefore, we can represent the deformation and deformation gradient schematically in the following diagram:

$$
X \in \kappa_{r}(\mathbb{B}) \underset{\xi=\chi\left(X, t_{0}\right)}{\longrightarrow} \xi \in \kappa_{t_{0}}(\mathbb{B}) \stackrel{I+H}{\boldsymbol{x}=\boldsymbol{\xi}+\boldsymbol{u}} \boldsymbol{x} \in \kappa_{t}(\mathbb{B})
$$




\subsection{Linearized constitutive equations}

We shall assume that the displacement gradient $H$ is small, $|H| \ll 1$, and linearize the constitutive equation (1) relative to the configuration $\kappa_{t_{0}}$, namely,

$$
T=T_{0}+\nabla_{F} \mathbb{F}_{\kappa_{r}}\left(F_{0}\right)\left[F-F_{0}\right]=T_{0}+\nabla_{F} \mathbb{F}_{\kappa_{r}}\left(F_{0}\right)\left[H F_{0}\right],
$$

or

$$
T=T_{0}+L\left(F_{0}\right)[H]
$$

where

$$
L\left(F_{0}\right)[H]=\nabla_{F} \mathbb{F}_{\kappa_{r}}\left(F_{0}\right)\left[H F_{0}\right]
$$

defines the fourth order elasticity tensor $L\left(F_{0}\right)$ relative to the reference configuration $\kappa_{t_{0}}$.

Furthermore, the (first) Piola-Kirchhoff stress tensor relative to the updated reference configuration $\kappa_{t_{0}}$ is given by

$$
\begin{aligned}
T_{\kappa_{t_{0}}} & =\operatorname{det}(I+H) T(I+H)^{-T} \\
& =\operatorname{det}(I+H)\left(T_{0}+L\left(F_{0}\right)[H]\right)(I+H)^{-T} \\
& =(I+\operatorname{tr} H)\left(T_{0}+L\left(F_{0}\right)[H]\right)\left(I-H^{T}\right)+o(2) \\
& =T_{0}+(\operatorname{tr} H) T_{0}-T_{0} H^{T}+L\left(F_{0}\right)[H]+o(2),
\end{aligned}
$$

where $o(2)$ represents higher order terms in the small displacement gradient $|H|$. We can also define the elasticity tensor for the Piola-Kirchhoff stress by

$$
L_{\kappa_{t_{0}}}\left(F_{0}, T_{0}\right)[H]=(\operatorname{tr} H) T_{0}-T_{0} H^{T}+L\left(F_{0}\right)[H],
$$

and write the linearized Piola-Kirchhoff stress as

$$
T_{\kappa_{t_{0}}}=T_{0}+L_{\kappa_{t_{0}}}\left(F_{0}, T_{0}\right)[H] .
$$

\subsection{Nearly incompressible elastic body}

For an incompressible elastic body, the constitutive equation (1) should be replaced by

$$
T=-p I+\mathbb{F}_{\kappa_{r}}(F), \quad \operatorname{det} F=1,
$$


where $p$ denotes the pressure which theoretically for a (perfectly) incompressible material body depends not only on the deformation of the body, but also on the boundary conditions.

However, we shall consider only nearly incompressible bodies, and assume that the pressure is a function of density, $p=p(\rho)$, or inversely, the density is a function of pressure, $\rho=\rho(p)$. Therefore, by nearly incompressible we mean that the density is nearly insensitive to the change of pressure, i.e., the density $\rho$ is nearly constant, i.e.,

$$
\frac{d \rho}{d p} \approx \text { const. } \ll 1 .
$$

In the approximation considered in (2), since $F=(I+H) F_{0}$, the Cauchy stress in the configurations $\kappa_{t}$ and $\kappa_{t_{0}}$ are given by

$$
T=-p I+\mathbb{F}_{\kappa_{r}}(F), \quad T_{0}=-p_{0} I+\mathbb{F}_{\kappa_{r}}\left(F_{0}\right),
$$

Linearization in $H$ with (4) gives

$$
T-T_{0}=-\left(p-p_{0}\right) I+\nabla_{F} \mathbb{F}_{\kappa_{r}}\left(F_{0}\right)\left[H F_{0}\right]=-\left(p-p_{0}\right) I+L\left(F_{0}\right)[H] .
$$

On the other hand, from the equation of mass balance, we have

$$
\dot{\rho}+\rho \operatorname{div} \dot{\boldsymbol{x}}=0,
$$

and since $\rho=\rho(p)$, it follows that

$$
\dot{p}+\beta \operatorname{tr} \operatorname{grad} \dot{\boldsymbol{x}}=0,
$$

where

$$
\beta=\rho\left(\frac{d \rho}{d p}\right)^{-1} \gg 1
$$

is a time-independent constant, much greater than 1 , by the assumption (8).

Furthermore, since grad $\dot{\boldsymbol{x}}=\dot{F} F^{-1}$ and $F=(I+H) F_{0}$, by assuming also that $|\dot{H}| \ll 1$, we have

$$
\operatorname{grad} \dot{\boldsymbol{x}}=\dot{H} F_{0}\left((I+H) F_{0}\right)^{-1}=\dot{H} F_{0} F_{0}^{-1}(I-H)+o(2)=\dot{H}+o(2),
$$

and the equation (10) becomes

$$
\dot{p}+\beta \operatorname{tr} \dot{H}=0,
$$


which by integration leads to

$$
p-p_{0}=-\beta \operatorname{tr} H .
$$

Consequently, from (9), the Cauchy stress becomes

$$
T=T_{0}+\beta(\operatorname{tr} H) I+L\left(F_{0}\right)[H],
$$

and from (6), the Piola Kirchhoff stress

$$
T_{\kappa_{t_{0}}}=T_{0}+\beta(\operatorname{tr} H) I+L_{\kappa_{t_{0}}}\left(F_{0}, T_{0}\right)[H] .
$$

Remark. Perhaps, someone might regard the parameter $\beta$ introduced in (11) as a penalization parameter. However, it is different from the usual penalization method which introduces a penalizing term into the momentum equation to account for kinematical or geometrical restrictions. In the present context, it is a consequence of near-incompressibility assumption (8) resulting in the elimination of the pressure from the constitutive equation by integrating the mass balance equation, and there is no need to modify the momentum equation.

\subsection{Mooney-Rivlin materials}

As an example, we shall consider a (nearly) incompressible Mooney-Rivlin material with the constitutive equation given by

$$
T=-p I+\mathbb{F}_{\kappa_{r}}(F), \quad \mathbb{F}_{\kappa_{r}}(F)=s_{1} B+s_{2} B^{-1},
$$

where $B=F F^{T}$ is the left Cauchy-Green strain tensor and the coefficients are constants.

From (4) and (5), after taking the gradient of $\mathbb{F}_{\kappa_{r}}(F)$ at $F_{0}$, we have

$$
L\left(F_{0}\right)[H]=s_{1}\left(H B_{0}+B_{0} H^{T}\right)-s_{2}\left(B_{0}^{-1} H+H^{T} B_{0}^{-1}\right),
$$

and

$$
\begin{aligned}
L_{\kappa_{t_{0}}}\left(F_{0}, T_{0}\right)[H]= & (\operatorname{tr} H) T_{0}-T_{0} H^{T}+s_{1}\left(H B_{0}+B_{0} H^{T}\right) \\
& -s_{2}\left(B_{0}^{-1} H+H^{T} B_{0}^{-1}\right) .
\end{aligned}
$$

In numerical examples presented later, the material is assumed to be of Mooney-Rivlin type and these relations are used. 


\section{Linearized boundary value problem}

We can now consider the updated Lagrangian formulation of the boundary value problem. Let $\Omega=\kappa(\mathbb{B}) \subset \mathbb{R}^{3}$ be the region occupied by the body at the configuration $\kappa$ (standing for $\kappa_{t_{0}}$ and instead of $\boldsymbol{\xi}$, use $\boldsymbol{X}$ as a typical point of $\kappa(\mathbb{B})$ for simplicity). $\partial \Omega=\Gamma_{1} \cup \Gamma_{2}$, and $\boldsymbol{n}_{\kappa}$ be the exterior unit normal to $\partial \Omega$. Let the displacement vector relative to the configuration $\kappa$ be

$$
\boldsymbol{u}(\boldsymbol{X}, t)=\boldsymbol{x}(\boldsymbol{X}, t)-\boldsymbol{X}, \quad \boldsymbol{x}\left(\boldsymbol{X}, t_{0}\right)=\boldsymbol{X} .
$$

Consider the boundary value problem of an elastic body in equilibrium without external body force given by

$$
\left\{\begin{array}{rll}
-\operatorname{Div} T_{\kappa}=0 & \text { in } & \Omega, \\
T_{\kappa} \boldsymbol{n}_{\kappa}=\boldsymbol{f} & \text { on } \quad \Gamma_{1}, \\
\boldsymbol{u}=\boldsymbol{g} & \text { on } \quad \Gamma_{2},
\end{array}\right.
$$

where the surface traction $\boldsymbol{f}\left(\boldsymbol{X}, t_{0}\right)$ and the displacement $\boldsymbol{g}\left(\boldsymbol{X}, t_{0}\right)$ are prescribed.

Assuming that at $t_{0}$, the deformation gradient $F_{0}$ with respect to the preferred configuration $\kappa_{r}$ and the Cauchy stress $T_{0}$ are known, and that $\Delta t=t-t_{0}$ is small enough, then from (13), we have

$$
T_{\kappa}=T_{0}+\beta(\operatorname{tr} H) I+L_{\kappa_{0}}\left(F_{0}, T_{0}\right)[H]:=T_{0}+\tilde{L}_{\kappa}\left(F_{0}, T_{0}\right)[H],
$$

where $H(\boldsymbol{X}, t)=\nabla \boldsymbol{u}(\boldsymbol{X}, t)$ is the displacement gradient with respect to $\boldsymbol{X}$ in the configuration $\kappa$. Upon substitution into (15), we have the following problem:

$$
\left\{\begin{aligned}
-\operatorname{Div}\left(\tilde{L}_{\kappa}\left(F_{0}, T_{0}\right)[\nabla \boldsymbol{u}]\right) & =\operatorname{Div} T_{0} & & \text { in } \quad \Omega, \\
\left(\tilde{L}_{\kappa}\left(F_{0}, T_{0}\right)[\nabla \boldsymbol{u}]\right) \boldsymbol{n}_{\kappa} & =\boldsymbol{f}-T_{0} \boldsymbol{n}_{\kappa} & & \text { on } \Gamma_{1}, \\
\boldsymbol{u} & =\boldsymbol{g} & & \text { on } \Gamma_{2} .
\end{aligned}\right.
$$

This is the same problem to determine the displacement vector $\boldsymbol{u}(\boldsymbol{X}, t)$ as a boundary value problem in linear elasticity. The present linearization does not rely on the incremental loading, as the driving force for the deformation of the body from the current state, used in the usual incremental methods (see $[4,7,6])$. 
At every time step, the idea of formulating the boundary value problem in the form (17) is similar to the theory of small deformations superposed on finite deformations (see $[2,1]$ ). In this manner, either we are interested in the evolution of solutions with gradually changing boundary conditions resulting in large deformation, or, we can treat the boundary values of finite elasticity as the final value of a successive small incremental boundary values at each time step.

Remark 3.1. The constant $\beta$ will be taken as a constant large enough to guarantee the near-incompressibility, which in numerical computation can be checked from the condition $\operatorname{det} F \approx 1$.

\section{Numerical solution for large deformations}

Recall the Euler method of solving differential equation, say $\dot{y}=f(t)$, that for a discrete time axis, $\cdots<t_{n-1}<t_{n}<t_{n+1}<\cdots$, and $y\left(t_{n}\right)=y_{n}$, the solution curve can be constructed by $y_{n+1}=y_{n}+f\left(t_{n}\right) \Delta t$, where $f\left(t_{n}\right)$ is the tangent of the solution curve at $t_{n}$. We can use a similar strategy for solving problems of large deformation, by solving linear boundary value problem stated in (17).

We consider a discrete time axis, $\cdots<t_{n-1}<t_{n}<t_{n+1}<\cdots$ with small enough constant spacing $\Delta t$. Let $\kappa_{t_{n}}$ be the configuration of the body at the instant $t_{n}$ and

$$
X=\boldsymbol{x}\left(\boldsymbol{X}, t_{n}\right) \in \kappa_{t_{n}}(\mathbb{B}) .
$$

Let

$$
F_{0}=F_{0}\left(\boldsymbol{X}, t_{n}\right), \quad T_{0}=T_{0}\left(\boldsymbol{X}, t_{n}\right)
$$

be the deformation gradient relative to the preferred configuration $\kappa_{r}$ and the Cauchy stress respectively. The boundary value problem (17) with boundary data $\boldsymbol{f}\left(\boldsymbol{X}, t_{n}\right)$ and $\boldsymbol{g}\left(\boldsymbol{X}, t_{n}\right)$ in Lagrangian formulation with respect to the reference configuration $\kappa_{t_{n}}$, can now be solved as a problem in linear elasticity for the displacement field $\boldsymbol{u}\left(\boldsymbol{X}, t_{n+1}\right)$ from the configuration $\kappa_{t_{n}}$.

After solving the problem (17) at $t_{n}$, the reference configuration $\kappa_{t_{n+1}}$ at $t_{n+1}$ can be updated from the displacement field, i.e.,

$$
\boldsymbol{x}\left(\boldsymbol{X}, t_{n+1}\right)=\boldsymbol{X}+\boldsymbol{u}\left(\boldsymbol{X}, t_{n+1}\right),
$$


while the deformation gradient

$$
F_{0}\left(\boldsymbol{X}, t_{n+1}\right)=\left(I+H\left(\boldsymbol{X}, t_{n+1}\right)\right) F_{0}\left(\boldsymbol{X}, t_{n}\right)
$$

and the Cauchy stress from (10)

$$
T_{0}\left(\boldsymbol{X}, t_{n+1}\right)=T_{0}\left(\boldsymbol{X}, t_{n}\right)+\beta \operatorname{tr} H\left(\boldsymbol{X}, t_{n+1}\right) I+L\left(F_{0}\left(\boldsymbol{X}, t_{n}\right)\right) H\left(\boldsymbol{X}, t_{n+1}\right)
$$

can be calculated at $t_{n+1}$ so that the Lagrangian formulation of the problem in the form (17), with boundary data $\boldsymbol{f}\left(\boldsymbol{X}, t_{n+1}\right)$ and $\boldsymbol{g}\left(\boldsymbol{X}, t_{n+1}\right)$, can proceed again from the updated reference configuration at $t_{n+1}$. This numerical procedure will be referred to as the successive Lagrangian formulation of linear approximation for large deformations, or simply as the method of Successive Linear Approximation (SLA).

Boundary value problems in finite elasticity known as Ericksen's problem are the textbook examples for which the exact solutions can be obtained by applying suitable surface tractions on the boundary alone irrespective of constitutive properties of the elastic bodies. Such controllable deformations are called universal solutions, and for any incompressible isotropic elastic bodies, there are several known classes of problems including homogeneous deformation, shearing, bending, torsion, inflation and eversion $[3,2]$. In developing a numerical method for large deformations, such problems with exact solutions can be used as benchmark problems for comparison. In the following we shall consider one such problem: pure shear of a square block.

\section{Example: pure shear}

For applying the method of SLA, we consider the case of pure shear of a square block, by applying tangential surface traction, the shear stress $\tau$, on the surface of the block as shown in Figure 1.

This boundary value problem requires that in the deformed state, the normal stresses must vanish at the boundary and the time-dependent shear stress be given by $\tau=\alpha t$ for some small constant $\alpha$ in order to be consistent with the assumption of small incremental deformation. In addition, since the boundary conditions are of the Neumann type, for uniqueness of solution in numerical computation, the point $O$ will be kept fixed and the line $\overline{O A}$ will be held in the horizontal direction as shown in Figure 1. 


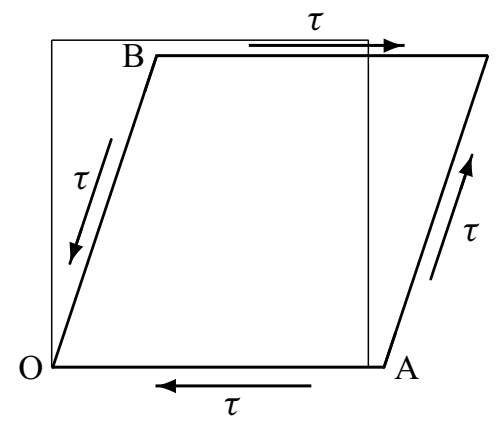

Figure 1 - Pure shear.

\subsection{Exact solution}

Theoretically, the shear deformation can be described as

$$
x_{1}=\lambda_{1} X_{1}+\kappa \lambda_{2} X_{2}, \quad x_{2}=\lambda_{2} X_{2}, \quad x_{3}=\lambda_{3} X_{3} .
$$

In the case of pure shear, for which there are no applied normal stresses on the surface, it can be proved (see for example, $[3,5]$ ) that for any elastic material body, the deformed state of a square block maintains the geometric shape of an equilateral parallelogram as shown in Figure 1, i.e.,

$$
\overline{O A}=\overline{O B}, \quad \text { or } \quad \lambda_{1}^{2}=\left(1+\kappa^{2}\right) \lambda_{2}^{2} .
$$

Furthermore, for Mooney-Rivlin materials, from (14) and (18), the shear stress $\tau=T_{12}$ is given by

$$
\tau=\kappa\left(s_{1} \lambda_{2}^{2}-s_{2} \lambda_{1}^{-2}\right) .
$$

In the following numerical computation, we shall consider the two-dimensional case, so that the thickness in $x_{3}$-direction remains unchanged, i.e.,

$$
\lambda_{3}=1, \quad \text { and } \quad \lambda_{1} \lambda_{2}=1,
$$

by incompressibility. Consequently, from (19) and (20), it follows that

$$
\lambda_{1}=\left(1-\tau^{2}\left(s_{1}-s_{2}\right)^{-2}\right)^{-1 / 4}, \quad \kappa=\left(\lambda_{1}^{4}-1\right)^{1 / 2} .
$$

Both the relations (19) and (21) will be used for comparison with the numerical results. 


\subsection{Numerical results}

We consider time step $t_{n}=n \Delta t$, so that the prescribed shear stress at time step $t_{n}$ is $\tau=n \Delta \tau$ for $\Delta \tau=\alpha \Delta t$. Following the method of SLA, at every step $n$, the reference stress $T_{0}$ and the deformation gradient $F_{0}$ are obtained from the previous step. We shall assume that the initial reference configuration $\kappa_{r}$ is stress-free, i.e., at $n=0$, we have $T_{0}=0$ and $F_{0}=I$.

The finite element method with initial rectangular bilinear elements is used and the following data are given for numerical computation: $s_{1}=1, s_{2}=-0.1$, $\Delta \tau=0.004$ and $\Omega=(0,1) \times(0,1), \Gamma_{1}=\partial \Omega$.
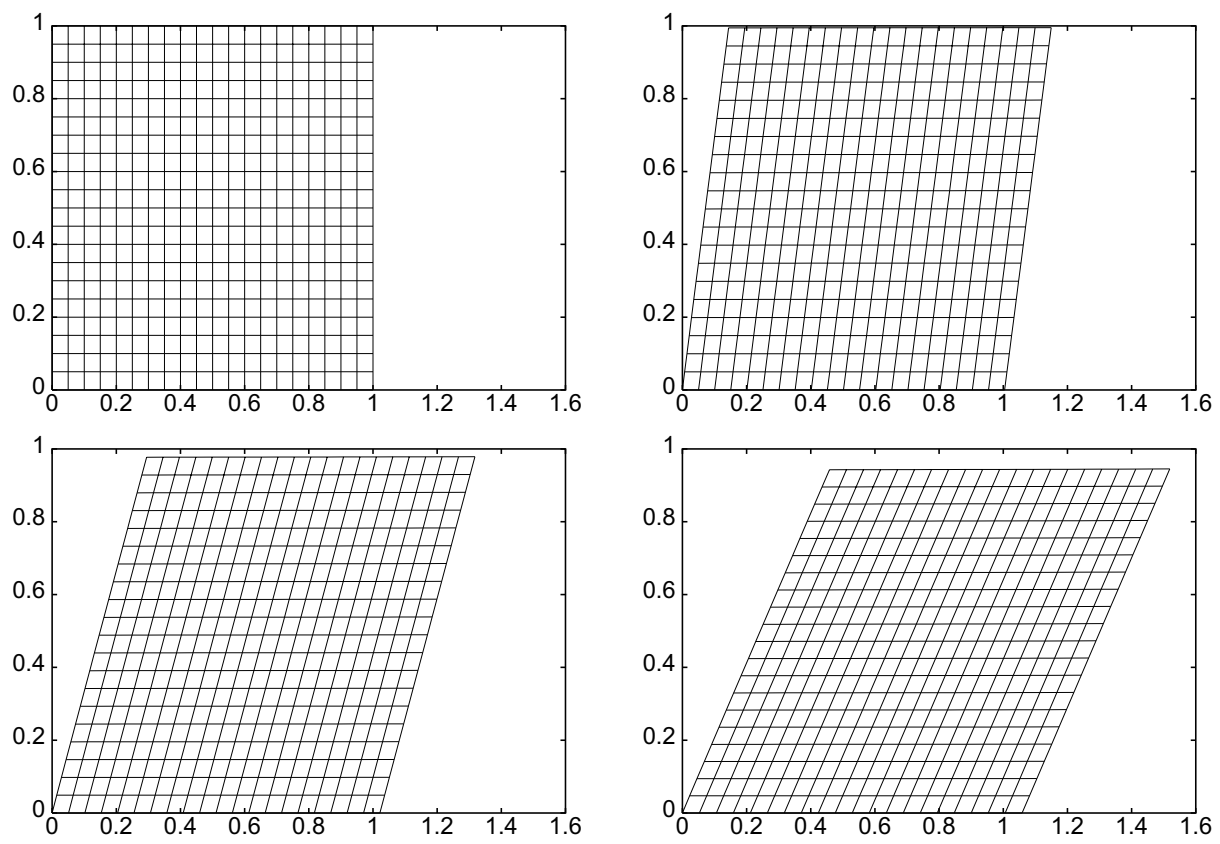

Figure 2 - The initial square (at $n=0$ ) and its subsequent deformed states at $n=40$, $n=80$, and $n=120$.

The numerical results are shown in Figure 2, in which the initial square and its deformed states at the applying shear stress $\tau=40 \Delta \tau, \tau=80 \Delta \tau$, and $\tau=120 \Delta \tau$ are shown. One can easily see that the geometric shapes of the deformed state are equilateral parallelograms as expected. Indeed, the errors for $n=40, n=80$, and $n=120$ are respectively of $0.07 \%, 0.09 \%$ and $0.99 \%$, 
by comparing the consecutive sides of the parallelogram, $(\overline{0 A}-\overline{0 B}) / \overline{0 A}$, using a mesh of $20 \times 20$ elements.

The deformation given by (18) is homogeneous, and the figures in Figure 2 seem to confirm this. In other words, theoretically the deformation gradient $F$ is constant. In particular, so are the values of $\lambda_{1}=F_{11}$ and $\operatorname{det} F$. These values have been checked, and surely, they are not expected to be exactly constant everywhere in the numerical results. Instead, we shall see how good those values are from being constant by showing their average values together with their minima and maxima over the body in Table 1 and Table 2 .

\begin{tabular}{ccccc}
\hline$n$ & $\lambda_{1}^{\mathrm{ex}}$ & $\lambda_{1}^{\mathrm{av}}$ & $\left(\lambda_{1}^{\min }-\lambda_{1}^{\mathrm{max}}\right)$ & Average Error \\
\hline 40 & 1.005360 & 1.005195 & $(1.003931-1.006040)$ & $0.0164 \%$ \\
80 & 1.022352 & 1.022929 & $(1.020991-1.024568)$ & $0.0564 \%$ \\
120 & 1.054227 & 1.060329 & $(1.056625-1.062738)$ & $0.5788 \%$ \\
\hline
\end{tabular}

Table 1 -Comparison of the exact value of $\lambda_{1}$ with the values obtained from numerical results at various step $n$.

\begin{tabular}{ccc}
\hline$n$ & $J^{\text {av }}$ & $\left(J^{\min }-J^{\max }\right)$ \\
\hline 40 & 1.000000 & $(0.999994-1.000001)$ \\
80 & 0.999996 & $(0.999988-0.999999)$ \\
120 & 0.999969 & $(0.999955-0.999976)$ \\
\hline
\end{tabular}

Table 2 - Verification of the incompressibility condition $J=\operatorname{det} F \approx 1$ in the numerical simulation at various step $n$.

At step $n$, the exact value of $\lambda_{1}$, given in Table 1 , is calculated from the equation (21) for the shear stress $\tau=n \Delta \tau$. The corresponding value obtained from the numerical computation is shown in the table. From the average value over the body and the percentage error, $\left|\lambda_{1}^{\text {av }}-\lambda_{1}^{\text {ex }}\right| / \lambda_{1}^{\text {ex }}$, it shows that it is in very good agreement with the analytical prediction. In addition, to show the value is nearly constant in the body, the minimum and the maximum values of $\lambda_{1}$ are also given.

From Table 2, the condition for near-incompressibility, $\operatorname{det} F \approx 1$, is clearly confirmed. The parameter $\beta=10^{4}$ has been taken in the numerical computation. 


\section{Final remarks}

For numerical computation of large deformations in finite elasticity, to obviate the difficulty of handling nonlinearities, "incremental methods" are widely discussed, for example, in the books by Oden [4], Ogden [7] and Ciarlet [6]. These methods consist in letting the boundary forces vary by small increments from zero to the given ones and to compute corresponding approximate solutions by successive linearization. The general idea seems to be of purely mathematical concern regarding linearization between successive boundary value problems. The problems are usually formulated with domains in the initial reference configuration, i.e., in Lagrangian formulation.

From mathematical viewpoint, the basic idea of the present method (SLA) is similar to the other incremental methods, except that it is formulated using the current state as the reference configuration and not relying on the incremental loading as the driving force for further deformations. Consequently, it would be possible to prove existence and uniqueness of successive incremental solutions with proper regularity conditions on the current state of the body (see for example [6]).

The conceptually simpler approach of the present method with successively updated Lagrangian formulation at each step is mainly motivated from physical viewpoint. It also has an advantage that the prescription of (incremental) boundary data is simpler and straightforward, if we remember that in Lagrangian formulation, the corresponding boundary tractions to be prescribed in the initial reference configuration generally depend on the surface geometry of the deformed configuration.

For example, in the problem of pure shear considered in this paper, knowing that the shear tractions have to be applied tangentially on the (slanted) surfaces of the deformed body (see Fig. 1), if one try to prescribe the boundary tractions on the initial undeformed body, one might encounter a difficulty that the magnitude as well as the direction of the tractions to be prescribed will also depend on the deformation of the surface which is itself unknown before the problem is solved, i.e., the boundary conditions depend on the solution itself, or at least, on the previous incremental solution. In the present method, however, it does not cause any difficulties at all, because in consecutive steps, it is a linear problem. 
Therefore, the difference between applying the corresponding tractions on the surfaces of the consecutive states is of higher orders which is insignificant in the linear approximation.

Another advantage of this formulation for (nearly) incompressible elastic body lies in the elimination of the pressure from the integration of the equation of mass balance. This is possible due to the assumption of near-incompressibility, so that unlike the usual formulation there is no need to consider the pressure as an unknown variable in addition to the unknown displacement vector function.

Acknowledgements. The authors (ISL and MAR) are partially supported by CNPq-Brasil.

\section{REFERENCES}

[1] A.E. Green, R.S. Rivlin and R.T. Shield, General theory of small elastic deformations superposed on finite deformations. Pro. Roy. Soc. London, Ser. A, 211 (1952), 128-154.

[2] C. Truesdell and W. Noll, The Non-Linear Field Theories of Mechanics. $3^{\text {rd }}$ edition. Springer: Berlin (2004).

[3] I-S. Liu, Continuum Mechanics. Springer: Berlin Heidelberg (2002).

[4] J.T. Oden. Finte Elements of Nonlinear Continua. McGraw-Hill, New York (1972).

[5] K.R. Rajagobal and A.S. Wineman, New universal relations for nonlinear isotropic elastic materials. J. Elasticity, 17 (1987), 75-83.

[6] P.G. Ciarlet, Mathematical Elasticity. Three-Dimensional Elasticity, 1, North-Holland, Amsterdam (1988).

[7] R.W. Ogden, Non-Linear Elastic Deformations. Ellis Horwood, New York (1984). 